\title{
Beamforming techniques for enabling spatial- reuse in MCCA 802.11s networks
}

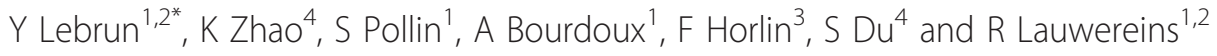

\begin{abstract}
We address the problem of co-channel interference (CCI) in wireless mesh networks based on the IEEE802.11s extension. The carrier sensing mechanism deployed in those networks insufficiently addresses the CCl problem, causing the hidden and exposed node problems; consequently degrading the throughput and latency. In this paper, we show how beamforming techniques can be implemented on top of the IEEE802.11s medium access control protocol and, using the information readily available, cancel the interference to mitigate this inefficiency of carrier sense and improve the spatial-reuse gain. In addition, we propose the signal-to-jamming-noise ratio (SJNR) beamformer and show that it significantly improves the spatial-reuse gain compared to the simple zero-forcing (ZF) beamformer and the basic IEEE802.11s access scheme. We derive the ergodic capacity of the ZF beamformer and the basic IEEE802.11s access scheme and simulate the performance of the various schemes. We show that improvements of up to $85 \%$ are achieved as function of the scenario simulated and the beamforming technique used and that the SJNR scheme outperforms the standard ZF beamformer.
\end{abstract}

Keywords: wireless mesh network (WMN), IEEE802.11s, beamforming, zero-forcing (ZF), signal-to-jamming-noise ratio (SJNR), spatial-reuse

\section{Introduction}

A wireless mesh network (WMN) based on the IEEE802.11s extension [1], as shown in Figure 1, can exploit neighbor nodes to relay the information through multiple hops in the network and increase the spectral and power efficiency. WMNs have recently been considered in wireless standards, e.g., the 802.15.5 [2] and the 802.16e [3], and are still seen as a promising research area in wireless communications. In such networks, an efficient spatial-reuse is imperative to maximize the use of the available spectrum and provide the required quality of service (QoS) in terms of throughput and latency [4]. Spatial-reuse means that multiple nodes communicate concurrently, using the same time/frequency resources. However, the medium access control (MAC) protocol of IEEE802.11s networks relies on carrier sensing for granting access to the medium. This carrier sense mechanism causes the hidden node problem, i.e., when a node that is able to interfere with an ongoing

\footnotetext{
* Correspondence: lebruny@imec.be

${ }^{1}$ Interuniversity Micro-Electronics Center (IMEC), Kapeldreef 75, 3001 Leuven, Belgium
}

Full list of author information is available at the end of the article transmission is not silenced, and the exposed node problem, i.e., when a node is silenced even when a transmission from this node does not cause a collision at the receiver. These problems are known to limit the spatial reuse, consequently degrading the performance of the network [5].

When sensing the medium as busy, nodes part of an IEEE802.11s network refrain from transmitting to prevent collisions at the receiver. Therefore, co-channel interference $(\mathrm{CCI})$ will considerably impact the transmit opportunities of the few relay stations (STAs) close the access point (AP) of a mesh network that aggregates most of its traffic towards these nodes, i.e., they will block each other when transmitting. To improve spatialreuse, it is then needed to allow relay STAs to transmit often (i.e., no exposed nodes) while avoiding interference from neighbor relay STAs (i.e., no hidden nodes). Achieving this in a distributed way is the ultimate goal of every distributed wireless system.

Many techniques have been proposed in the literature to mitigate these problems, ranging from contention window adaptation, transmit power control [6], tuning of the threshold [7] to rate adaptation [8] and routing 


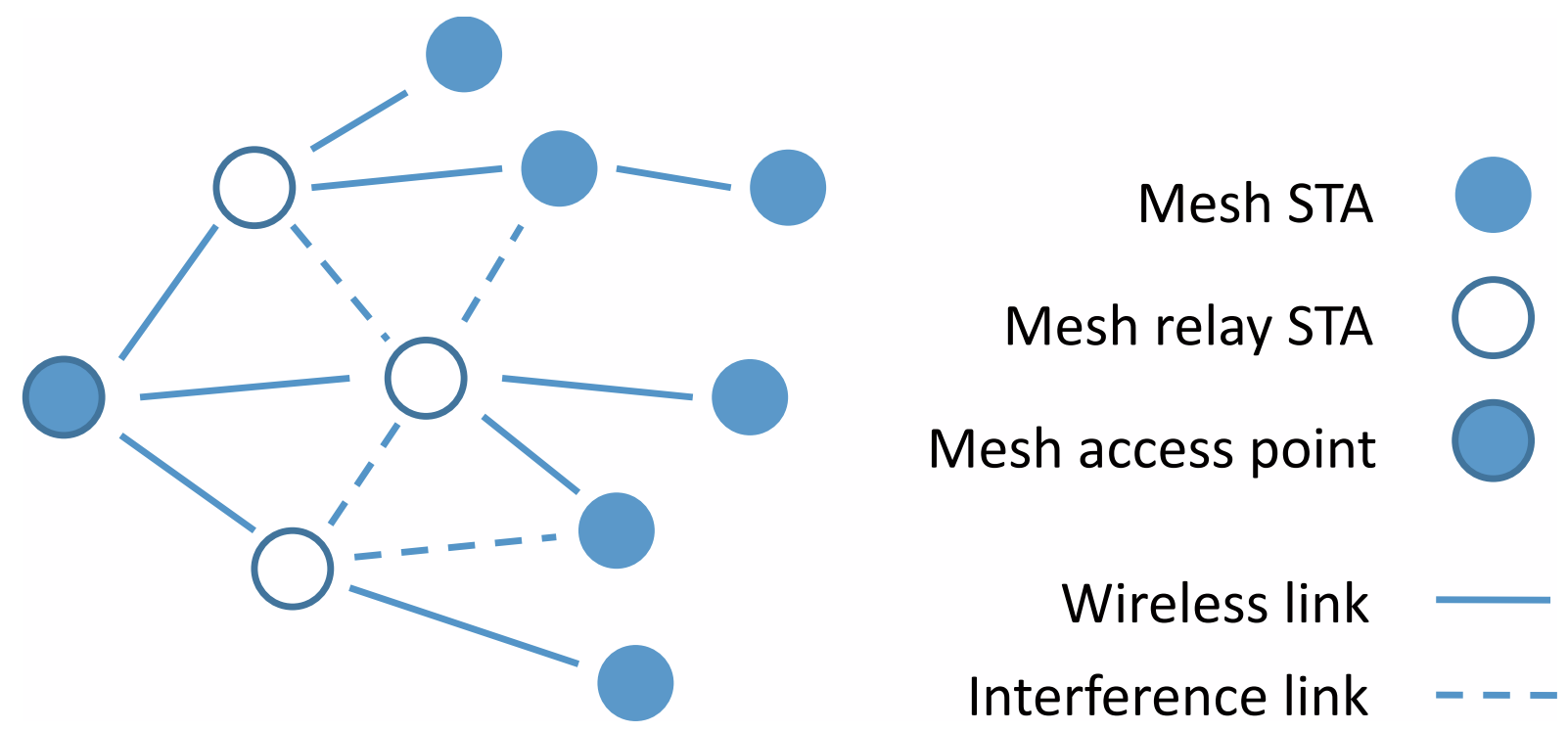

Figure 1 In this paper, we propose a new solution to improve the performance of mesh networks. This is achieved by solving the CCl problem by coupling the MAC protocol with distributed beamforming. As a result, the relaying mesh station that was blocked, i.e., because of the interference link, is now allowed to transmit. We show that significant spatial-reuse gains can be achieved depending on the scenario and the beamforming technique used. For the beamforming, a new scheme is proposed that outperforms the standard ZF beamformer and the basic access scheme.

[9]. All techniques aim at balancing the negative impact of the exposed node versus the hidden node problems. For example, an increase in transmit power improves the energy received at the receiver and silences more nodes (increases the blocking area) hence decreasing the number and impact of hidden nodes collisions. However, this comes at the cost of a higher number of exposed nodes hence degrading the spatial-reuse gain. In [10], it is shown that the exposed node problem, when relying on distributed resource allocation, should not be avoided but that there is an optimal trade-off between the two problems. No MAC-layer techniques only is capable of removing the inefficiencies of the hidden versus exposed node problems.

In addition, PHY-layer techniques may be used to cancel the interference and prevent a collision at the receiver [11-13]. For example, zero-forcing (ZF) beamforming for interference cancellation has been shown to increase the capacity of ad-hoc networks [14]. Beamforming is indeed a promising approach to mitigate the negative impact of the CCI, i.e., the concurrent node may transmit even though it senses the channel as busy. However, to apply the optimal weights on each antenna and cancel interference, these techniques require the perfect channel state information (CSI) between the transmitter and the ongoing and targeted nodes. This is difficult to implement in such distributed networks and requires an adaptation of the MAC protocol $[15,16]$. Alternatively, techniques exist that rely on partial CSI that is obtained by the request to send/clear to send (RTS/CTS) frames, e.g., the circular transmissions of the RTS frames [17]. These schemes that rely instead on sub-optimal beamforming or imperfect CSI hence provide not-optimal performance. In [18], the RTS/CTS frames are used to acquire the partial CSI and focus the energy towards the targeted receiver, instead of canceling the $\mathrm{CCI}$ this increases the throughput and mitigates the hidden node problem, e.g., the receiver is more resilient to interference. Such a scheme can also be used to reduce the transmit power while achieving the same performance hence reducing the generated interference and mitigating part of the exposed node problem [19]. Alternative methods to obtain imperfect CSI, e.g., estimation of the location from GPS or the angle of arrival, have also been proposed but provide also sub-optimal performance [20]. Moreover, in addition to the CSI, precise timing information is needed at the concurrent transmitter for synchronization, i.e., the timing information of the user it does not harm. Furthermore, the communication protocol may use an acknowledgment (ACK) frame to confirm the successful transmission, this is a possible source of collisions. Implementation of beamforming techniques is hence promising but challenging to achieve in practical scenarios.

To conclude, mitigating the negative impact of $\mathrm{CCI}$ is key to improve the number of spatial-reuse opportunities in the IEEE802.11s network and provide the required QoS. As introduced above, there is a 
fundamental trade-off between the exposed and hidden node problems and several MAC-layer techniques have been proposed to tackle it. However, these techniques do not achieve optimal performance. A further step consists then in exploiting PHY-layer techniques, i.e., beamforming, to apply weights on each transmit antenna to mitigate the interference and maximize the spatial-reuse. In centralized networks, the timing, channel and data information are available at the central coordinator which can then share such information with selected users to enable concurrent or cooperative transmissions. This is, e.g., the case with the coordinated multipoint (CoMP) technique in LTE-advanced systems [21]. However, in distributed networks the sharing of information is difficult because of the lack of coordination among the users. The challenge lies then in acquiring the channel and synchronization information in such a decentralized network without change in the MAC protocol.

In this paper, we show how beamforming techniques can be implemented on top of the mesh coordinated channel access (MCCA) IEEE802.11s MAC protocol and, using the information readily available, improve the capacity and latency of such networks (the generalization of the proposed method to any distributed protocol is hence not possible). Secondly, we propose the signalto-jamming-noise ratio (SJNR) beamformer to balance the interference and signal quality of the intended receiver, and show that it significantly improves the spatialreuse gain compared to the simple ZF beamformer and the basic IEEE802.11s access scheme. The specific scenario that we consider for the performance analysis is an IEEE802.11s network, composed of two relaying stations source of most of the traffic and close to each other, hence blocking each other's channel access when transmitting if no precautions are taken.

The overview of the IEEE802.11s and the MAC MCCA mechanisms to access the channel are given in Section 2; the concrete scenario and goal of the study is then presented in Section 3. Section 4 presents the system model and the derivations of the ergodic capacity for the considered system with the basic IEEE802.11s and the ZF schemes and introduce the SJNR beamformer (Section 4-D). Simulations in Section 5 show the performance of the different schemes. These results are discussed together with the proposed analytical derivations. Section 6 concludes our paper.

We use the following notations. The vectors and matrices are in boldface letters, vectors are denoted by lower-case and matrices by capital letters. The superscript $(\cdot)^{H}$ denotes the Hermitian transpose operator and $(\cdot)^{\dagger}$ denotes the pseudo-inverse, $E[\cdot]$ is the expectation operator. $\mathrm{I}_{N}$ is an identity matrix of size $(N \times N)$ and $\mathbb{C}$ $N \times 1$ denotes the set of complex vectors of size $(N \times 1)$. The definition $\mathbf{x} \sim \mathbb{C} N\left(\mathbf{0}, \sigma^{2} \mathrm{I}_{N}\right)$ means that the vector $\mathbf{x}$ of size $N \times 1$ has zero-mean Gaussian distributed independent complex elements with variance $\sigma^{2}$. We define $\mathbf{a}^{n}$ as the $n$th element of the vector $\mathbf{a}$.

\section{Background: IEEE802. 11s and MCCA mechanism}

The IEEE802.11s is an amendment to the IEEE802.11 standard that specifies the physical -and MAC-layer specifications for enabling mesh networking for WLANs. Devices within such a network can exploit multi-hop communications to relay the information cleverly in the network as illustrated in Figure 1.

Access to the channel is handled by the mesh coordination function (MCF) which consists of the EDCA, a QoS-enhanced version of the well-known basic distributed coordination function (DCF), and the optional MCCA protocols. In this work, focus is on the MCCA protocol and the information sharing it facilitates. The MCCA is a scheduled resource allocation method, in which the schedule is determined in a distributed way. It results in contention-free communications in contrast with the EDCA mechanism. The schedule allows to determine and learn about transmissions in advance, which facilitates distributed beamforming techniques that require such coordination among the different transmitters. Below, the beaconing and reservation protocol are detailed.

In such network, the mesh stations use the enhanced distributed channel access (EDCA) or the optional mesh coordinated channel access (MCCA) mechanisms to access the channel. Although those modes differ, they both rely on carrier sensing for granting access to the channel. The EDCA scheme is a contention-based mechanism which itself is an improved variant of the basic IEEE802.11 DCF. Implementing spatial-reuse for such a mode is challenging and would require prior cooperation between the mesh stations. On the other hand, the MCCA mechanism is a non-contention-based process where the transmit opportunities (TXOP) are allocated in the future. Because each STA advertises its reserved TXOPs, both the CSI and the timing information for enabling beamforming may be obtained.

\section{A. Beaconing and synchronization}

With the MCCA mechanism, STAs broadcast beacon and delivery traffic indication messages (DTIM) frames on a periodic basis. These frames are used for advertising the scheduled transmissions and synchronization purpose, e.g., for the STAs to detect and join the network. In addition, to prevent a STA outside the beacon range to conflict with existing scheduled transmissions, STAs include the transmit opportunities of their neighbors in their beacon and DTIM frames. Nearby mesh STAs listen then to these frames to update their 


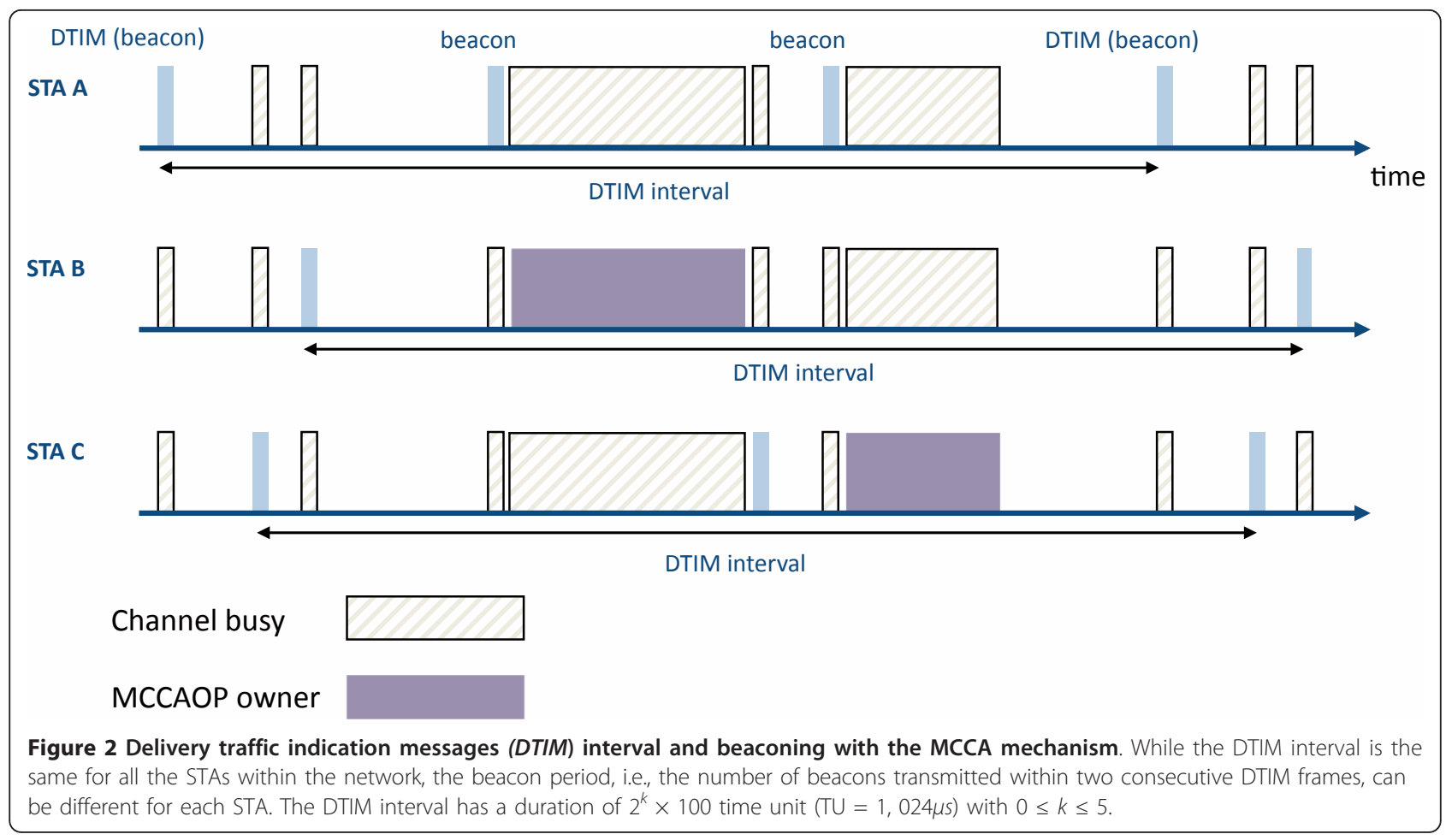

network allocation vector (NAV) accordingly. The NAV works as a virtual carrier sensing and indicates the scheduled transmissions and hence the duration for which a STA must defer from accessing the channel. Figure 2 shows an example of the beacon and DTIM frames structure.

\section{B. Distributed reservation protocol}

The optional medium access protocol called MCCA is a distributed reservation mechanism that allows mesh stations to avoid frame collisions by reserving transmit opportunities in the future, called MCCA opportunities (MCCAOPs). The handshake process is detailed in Figure 3. Most importantly, the MCCAOP contains detailed timing information such as the start and duration of the intended transmission. Nodes overhearing the handshake will hence know that information and be able to use it. In addition, nodes overhearing the MCCAOP Setup Reply from the intended receiver will be able to determine an estimate of the channel between themselves and that intended receiver. As a result, both timing and CSI informations are available and can be used by the physical layer beamformer to mitigate interference.

The MCCAOP control frames are transmitted when no MCCAOPs have been scheduled. The mesh STAs compete then to access the medium using the basic EDCA mechanism and gain access to the medium if it senses the channel idle for a duration in line with the
EDCA access category. At the beginning of an MCCA reservation, the STAs other than the MCCAOP owner refrain from accessing the channel. In this paper, the goal is to study the spatial-reuse opportunities during the planned MCCAOP, which means, studying if it is feasible to access the channel simultaneously without causing severe interference to the receiver. This minimal interference should be realized by implementing a (distributed) beamforming scheme using information that is available after the first MCCAOP establishment. No extra MAC layer overhead should be added, and the spatial-reuse gains realized should hence be net and realized above the MAC layer with its associated overhead.

\section{Scenario and problem formulation}

We propose how to combine advanced distributed beamforming techniques at physical layer to increase the overall network capacity. We show how these techniques can be implemented on top of the IEEE802.11s MAC protocol and the information available from the MCCA mechanism.

The scenario of interest consists of an IEEE802.11s system where the coverage areas of two relay STAs overlap. Because the IEEE802.11s system relies on (virtual) carrier sensing for accessing the channel, the two relays then block each other's transmissions; consequently decreasing the network capacity. To measure the negative impact of blocked transmissions, we first derive the probability for a relay to sense the channel as 


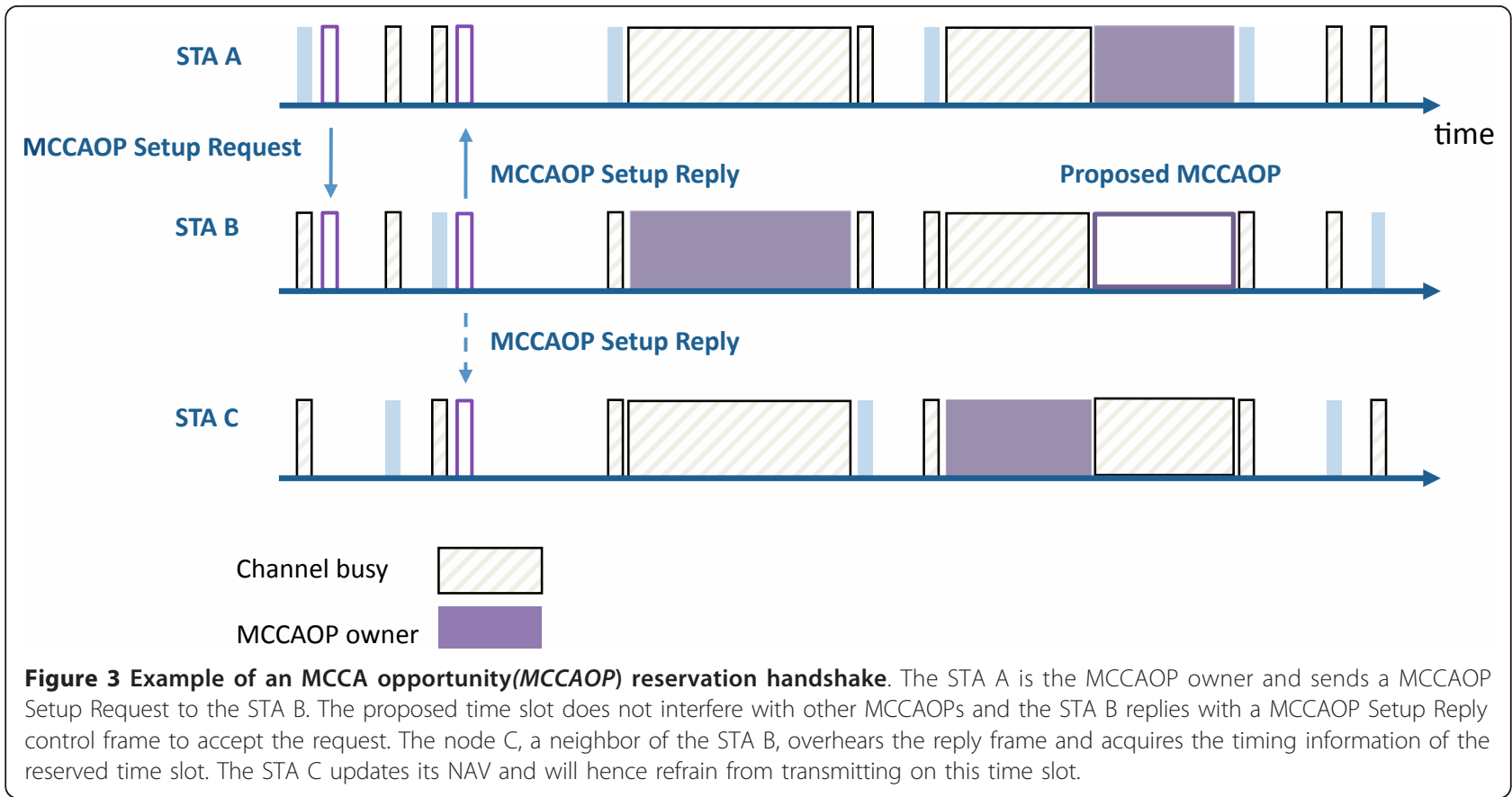

busy and block its transmission (Section 3-A). Next, we describe how beamforming techniques could be implemented to maximize the spatial-reuse in an IEEE802.11s using the MCCA mechanism and hence decrease the blocking probability in Section 3-B. However, decreasing that probability comes at a cost of increased interference, as function of the beamformer used, as will be explained in the next Section of the paper.

\section{A. Probability of interfering}

The system runs in time division multiple access (TDMA) and is composed of MCCA capable devices only with the assumption of heavy load. Figure 4 shows an example of the considered scenario. The amount of blocked transmissions in the network depends on the size of the overlapping area $\left(A_{I}\right)$, hence on the coverage radius $r_{i}$ of each relay and the distance $d$ between them (units are in meters). We express the overlapping area $A_{I}$ as

$$
A_{l}=r_{1}^{2} \cos ^{-1}\left(\frac{x}{r_{1}}\right)+r_{2}^{2} \cos ^{-1}\left(\frac{d-x}{r_{2}}\right)-r_{1} x \sqrt{1-\left(\frac{x}{r_{1}}\right)^{2}}-r_{2}(d-x) \sqrt{1-\left(\frac{d-x}{r_{2}}\right)^{2}}
$$

and

$$
x=\frac{r_{1}^{2}+d^{2}-e_{2}^{2}}{2 d} .
$$

In the extreme case where the coverage area of a Relay $_{k}$ is fully within the coverage area of the second Relay $l_{l}$ i.e., $d^{2}<\left(r_{k}-r_{l}\right)^{2}$, the overlapping area is equal to the coverage area of the Relay $y_{k}$ and $A_{I}=\pi r_{k}^{2}$.

Assuming uniformly distributed STAs, we then measure the probability for the relays to sense the channel as busy and be blocked. The probability of the $i$ th relay STA to be blocked is given as $p\left(T_{i}\right)=\frac{1}{2} \frac{A_{I}}{C_{i}}$ where $C_{i}$ denotes the coverage area of the $i$ th relay STA, i.e., $\pi r_{i}^{2}$. For example, for a system with $r_{1}=90, r_{2}=80$ and $d=$ 100 , the overlapping area is $A_{I}=6700$. From Equation (1) and Equation (2) we obtain
$p\left(T_{1}\right)=\frac{1}{2} \frac{A_{I}}{C_{1}}=\frac{3360}{\pi 90^{2}}=0.132$ and $p\left(T_{2}\right)=\frac{1}{2} \frac{A_{I}}{C_{2}}=0.167$.

\section{B. Feasibility of spatial-reuse}

In the following, we define as a primary relay ( Relay $_{\mathrm{i}}$ ) the first relay to gain access to the channel and as a primary STA $\left(\mathrm{STA}_{1}\right)$ its associated receiver. Similarly, Relay $_{2}$ denotes the blocked (or concurrent) relay and $\mathrm{STA}_{2}$ its associated receiver. As introduced in Section 2$\mathrm{B}$, the transmit opportunities are reserved through a handshake process. Because the two relays coexist, such a handshake may happen between a relay and a STA located in the overlapping area of the two relays. In this situation, the Relay 2 overhears the MCCAOP Setup Reply frame and hence learn the timing information of the scheduled transmission and estimates the channel between itself and this primary receiver. Then, following the IEEE802.11s protocol it refrains from transmitting on this MCCAOP (Section 2).

However, if equipped with multiple antennas, the Relay $_{2}$ may apply beamforming weights to enable concurrent transmissions. By exploiting the reciprocity of the channels from the MCCAOP Setup Reply frame, it can exploit its estimate of the channel to mitigate 


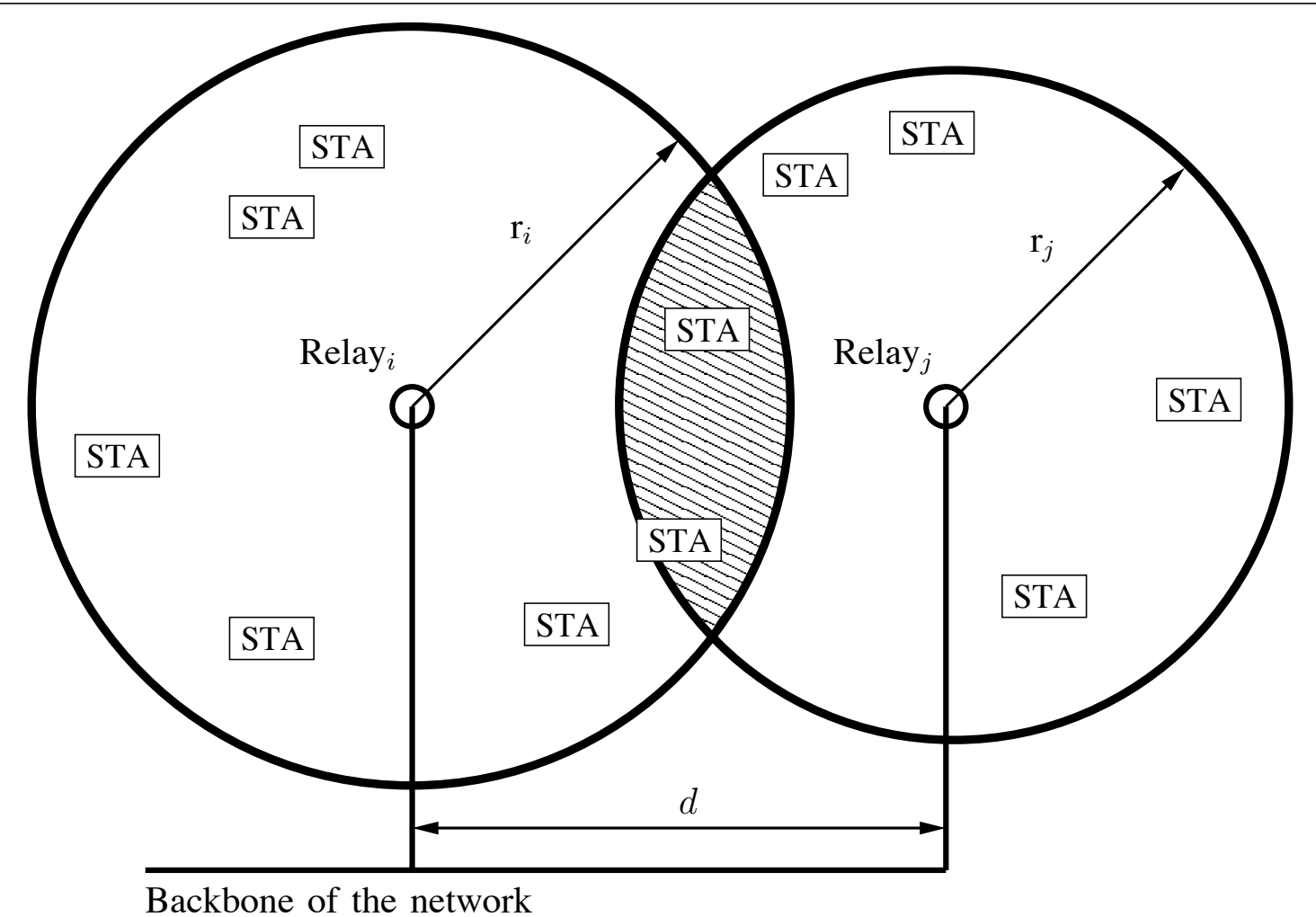

Figure 4 Example of an IEEE802.11s mesh network. The variable $d$ denotes the distance between the two relay stations and $r_{i}$ is the

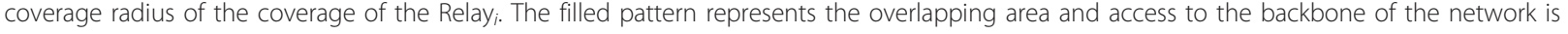
handled through a wired or a wireless link.

interference towards $\mathrm{STA}_{1}$ while communicating with $\mathrm{STA}_{2}$; consequently improving the spectral efficiency.

The Relay 2 begins then a reservation process with a selected $\mathrm{STA}_{2}$ for the same MCCAOP as the primary transmission. Because this request process conflicts with the existing MCCAOP, the Relay ${ }_{2}$ modifies the NAVs of the nearby STAs (including $\mathrm{STA}_{1}$ and $\mathrm{STA}_{2}$ ) to allow the spatial-reuse, i.e., a single additional field in the MCCAOP control frames is needed compared with the existing scheme.

\section{Transmit beamforming for spatial-reuse}

In this Section, we propose the system model (4-A) and the derivations of the ergodic capacity, i.e., the timeaveraged capacity of a stochastic channel, of the considered system with the basic IEEE802.11s and the ZF beamformer (Section 4-B and 4-C). In Section 4-D, we introduce the proposed SJNR beamformer.

\section{A. System model}

Each relay STA is equipped with multiple antennas $\left(N_{t}\right.$ $\geq 2$ ) while each STA has just a single antenna. The primary relay STA ( Relay $_{1}$ ) does not generate interference to the concurrent STA $\left(\mathrm{STA}_{2}\right)$. On the other hand, the concurrent transmitter $\left(\right.$ Relay $\left._{2}\right)$ interferes with the primary STA $\left(\mathrm{STA}_{1}\right)$. Figure 5 shows the considered scenario.

We consider flat fading channels and denote as a direct-link the channel vector between a relay STA and its dedicated STA. That is, the channel vector $\mathbf{h}_{1}^{H}$ for Relay $_{1}$ and $\mathbf{h}_{2}^{H}$ for Relay $y_{2}$. Similarly, we define the crosslink, i.e., $\mathbf{h}_{c l}^{H}$, as the channel vector between the Relay 2 and $\mathrm{STA}_{1}$. The direct-link channel vectors have independent and identically distributed (i.i.d.) elements of zero-mean and unit variance, $\mathbf{h}_{i}^{H} \sim \mathbb{C N}\left(\mathbf{0}, \mathbf{I}_{N_{t}}\right)$. The cross-link channel vector have i.i.d. elements of zeromean and variance $\sigma_{c l}^{2}, \mathbf{h}_{c l}^{H} \sim \mathbb{C N}\left(\mathbf{0}, \sigma_{c l}^{2} \mathbf{I}_{N_{t}}\right)$. As introduced above, Relay, has the knowledge of both the direct and the cross-link channels, i.e., $\mathbf{h}_{2}^{H}$ and $\mathbf{h}_{c l}^{H}$. Relay $_{1}$ has the knowledge of the channels from its antennas to $\mathrm{STA}_{1}$, i.e., $\mathbf{h}_{1}^{H}$. The CSI is obtained from the MCCAOP replies during the handshake process or through the beacon transmissions. The transmitted vector of the beamforming scheme, at Relay ${ }_{i}$, is denoted by $\mathbf{x}_{i} \in \mathbb{C}^{N_{t} \times 1}$. and can be expressed as follows

$$
\mathbf{x}_{1}=s_{1} \mathbf{w}_{1} \text { and } \mathbf{x}_{2}=s_{2} \mathbf{w}_{2}
$$




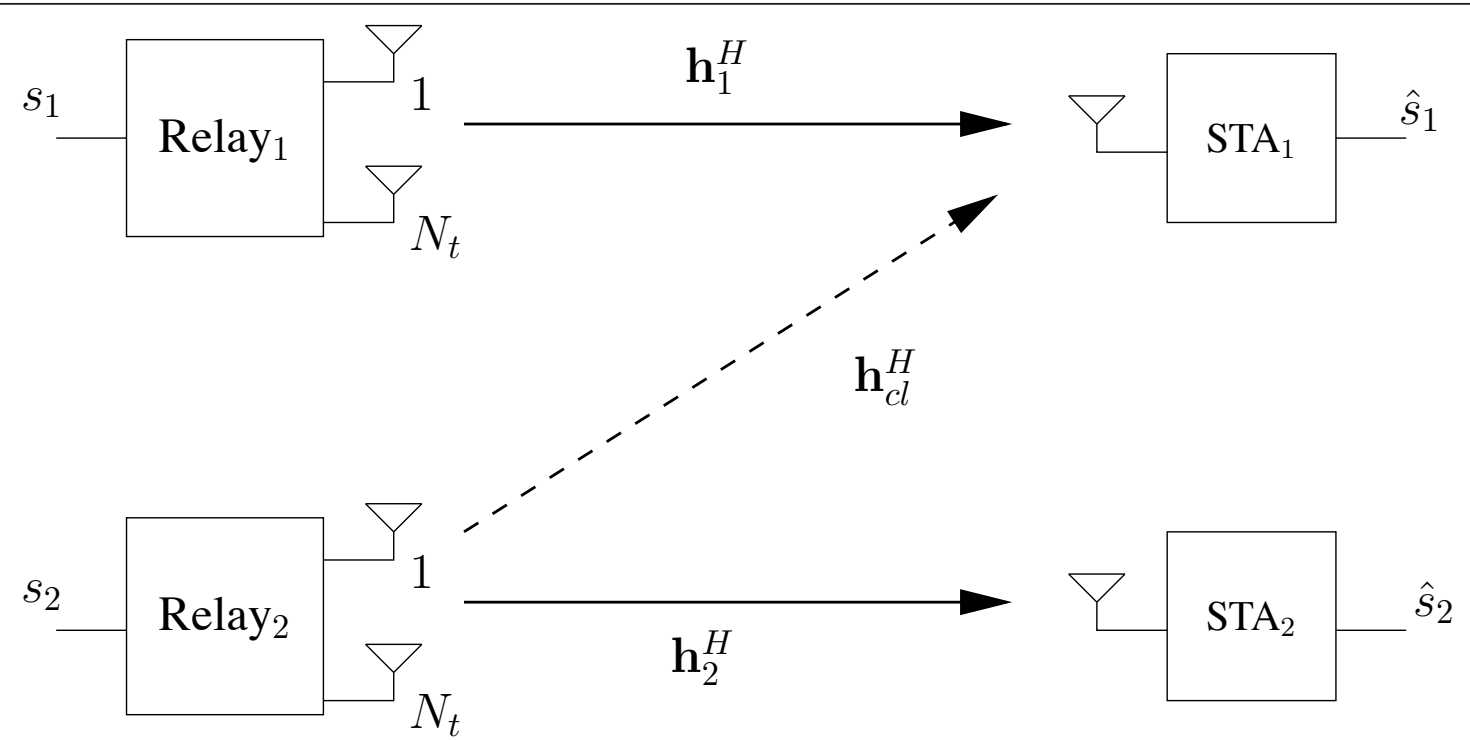

Figure 5 System model of the considered scenario in flat fading channels where both relay STAs communicate simultaneously toward their target STA. In this scenario, Relay ${ }_{2}$ creates interference towards the primary STA (STA $)$.

where $s_{i} \in \mathbb{C}^{1 \times 1}$ denotes the symbol transmitted by Relay $_{i}$ such that $E_{s}\left[s_{i} s_{i}^{H}\right]=1$; and $\mathbf{w}_{i} \in \mathbb{C}^{N_{t} \times 1}$ is the beamforming vector at Relay ${ }_{i}$ subject to the power constraint

$$
\mathbf{w}_{i}^{H} \mathbf{w}_{i} \leq P_{i} \quad i=1,2 .
$$

At the channel output, the received signal at the STA $i$ is denoted by $y_{i} \in \mathbb{C}^{1 \times 1}$

$$
\begin{aligned}
& y_{1}=\mathbf{h}_{1}^{H} \mathbf{w}_{1} s_{1}+\mathbf{h}_{c l}^{H} \mathbf{w}_{2} s_{2}+n_{1} \\
& y_{2}=\mathbf{h}_{2}^{H} \mathbf{w}_{2} s_{2}+n_{2} .
\end{aligned}
$$

In Equation (5), the first term denotes the desired signal, the second term represents the interference and the third term $n_{i} \in \mathbb{C}^{1 \times 1}$ is the additive white Gaussian noise (AWGN) with variance $\sigma_{n}^{2}$. The concurrent node $\mathrm{STA}_{2}$ is outside the range of the Relay ${ }_{1}$ and hence does not suffer from interference.

\section{B. Basic IEEE802.11s, no spatial-reuse}

Because the IEEE802.11s basic access scheme will not allow concurrent transmissions in the presence of CCI, the interference term in Equation (5) can hence be removed, i.e., $y_{1}=\mathbf{h}_{1}^{H} \mathbf{w}_{1} s_{1}+n_{1}$. Next, assuming a zeroforcing equalizer at the receiver, after processing, the estimated symbol can be expressed as $y_{i}=s_{i}+\left(\mathbf{h}_{i}^{H} \mathbf{w}_{i}\right)^{\dagger} n_{i}$. We then derive the instantaneous SNR $(\gamma)$ by taking the expectations over the noise and the symbols, i.e.,

$$
\gamma_{i}=\frac{E\left[s_{i} s_{i}^{H}\right]}{\left(\left(\mathbf{h}_{i}^{H} \mathbf{w}_{i}\right)^{H} \mathbf{h}_{i}^{H} \mathbf{w}_{i}\right)^{-1} E\left[n_{i} n_{i}^{H}\right]} .
$$

Given $E\left[n_{i} n_{i}^{H}\right]=\sigma_{n}^{2}$, the inverse term being a scalar, we can then write

$$
\gamma_{i}=\frac{1}{\sigma_{n}^{2}}\left(\mathbf{h}_{i}^{H} \mathbf{w}_{i}\right)^{2} .
$$

The Relays use the transmit maximum-ratio combining (transmit MRC) beamformer towards the targeteduser [22]. The weights of the transmit MRC beamformers are given as

$$
\mathbf{w}_{i}=\frac{\sqrt{P_{i} \mathbf{h}_{i}}}{\sqrt{\mathbf{h}_{i}^{h} \mathbf{h}_{i}}}
$$

where $\mathrm{w}_{i}$ satisfies the power constraint in (4). As a result we have $\mathbf{h}_{i}^{H} \mathbf{w}_{i}=\sqrt{P_{i} \sum_{n=1}^{N_{t}}\left|\mathbf{h}_{i}^{n}\right|^{2}}$.

We then express the ergodic capacity in bit/seconds/ Hertz (bps/Hz) for the data transmission $C_{E}$, where the ergodic capacity gives an upper bound of the average capacity [23], i.e.,

$$
E\left[\log _{2}(1+\gamma)\right] \leq \log _{2}(1+E[\gamma]) .
$$

We can then express $C_{E}$ as

$$
\begin{aligned}
& \log _{2}\left(1+\frac{1}{\sigma_{n}^{2}} E\left[\left(\mathbf{h}_{1}^{H} \mathbf{w}_{1}\right)^{2}\right]\right)\left(1-p\left(T_{1}\right)\right)+\log _{2}\left(1+\frac{1}{\sigma_{n}^{2}} E\left[\left(\mathbf{h}_{2}^{H} \mathbf{w}_{2}\right)^{2}\right]\right)\left(1-p\left(T_{2}\right)\right) \\
& =\log _{2}\left(1+\frac{P_{1}}{\sigma_{n}^{2}} \sum_{n=1}^{N_{4}} E\left[\left[\left.\mathbf{h}_{1}^{n_{1}}\right|^{2}\right]\right)\left(1-p\left(T_{1}\right)\right)+\log _{2}\left(1+\frac{P_{2}}{\sigma_{n}^{2}} \sum_{n=1}^{N_{4}}\left[\left|\mathbf{h}_{2}^{n_{2}}\right|^{2}\right]\right)\left(1-p\left(T_{2}\right)\right) .\right.
\end{aligned}
$$


The expression $\left|\mathbf{h}_{i}^{n}\right|^{2}$ follows a Chi-square distribution [24], we hence obtain

$$
E\left[\sum_{n=1}^{N_{t}}\left|\mathbf{h}_{i}^{n}\right|^{2}\right]=N_{t} .
$$

We can write the ergodic capacity of the data transmission for the basic 802.11s scheme as

$$
\left.C_{E}=\log _{2}\left(1+\frac{1}{\sigma_{n}^{2}} N_{t}\right)(1-p)\left(T_{1}\right)\right)+\log _{2}\left(1+\frac{1}{\sigma_{n}^{2}} N t\right)\left(1-p\left(T_{2}\right)\right)
$$

For example, the ergodic capacity for a $20 \mathrm{~dB}$ signal tonoise ratio (SNR), $r_{1}=90, r_{2}=80$ and $d=100$ with $N_{t}$ $=2$ and $P_{1}=P_{2}=1$, where $p\left(T_{1}\right)=\frac{1}{2} \frac{A_{I}}{C_{1}}=0.132$ and $p\left(T_{2}\right)=\frac{1}{2} \frac{A_{I}}{C_{2}}=0.167$ (Section 3-A). We then have

$$
C_{E}=\log _{2}\left(1+100 N_{t}\right) 0.87+\log _{2}\left(1+100 N_{t}\right) 0.833=13 \mathrm{bps} / \mathrm{Hz} .
$$

\section{Spatial-reuse with ZF beamforming}

In such a mode, when a relay STA senses the channel as busy, it employs the zero-forcing beamformer to cancel interference towards the primary STA while maximizing the energy towards the concurrent STA using the remaining degrees of freedom available.

1) Null beamforming: To cancel the interference towards $\mathrm{STA}_{1}$, the matrix $\mathrm{Z} \in \mathbb{C}^{N_{t} \times N_{t}}$ is used as the orthogonal projection onto the orthogonal complement of the column space of the channel $\mathrm{h}_{c l}$; from Relay 2 to cancel interference towards the primary $\mathrm{STA}_{i}$

$$
\mathbf{Z}=\mathbf{I}_{N_{t}}-\mathbf{h}_{c l}\left(\mathbf{h}_{c l}^{H} \mathbf{h}_{c l}\right)^{-1} \mathbf{h}_{c l}^{H}
$$

2) Maximum-ratio combining: the transmit-MRC beamformer is applied towards the targeted-user. The weights are chosen from the complementary space of the projection matrix to maximize the energy towards the concurrent $\mathrm{STA}_{2}$

$$
\mathbf{w}_{2}=\sqrt{P_{2}} \frac{\mathbf{Z h}_{2}}{\left\|\mathbf{Z} \mathbf{h}_{2}\right\|}
$$

which fulfills the power constraint in (4). Since the ZF beamforming weights lay in the null space of the nontargeted user, the received signal is interference free, Equation (5) can be written as $\gamma_{1}=\mathbf{h}_{1}^{H} \mathbf{w}_{1}+n_{1}$. We have expressed the transmit and received signals and defined the beamforming weights for the considered scheme. Next, from the results in (16), the combination of the precoder with the channel $\mathbf{h}_{2}^{H} \mathbf{w}_{2}$, gives

$$
\mathbf{h}_{2}^{H} \mathbf{w}_{2}=\sqrt{P_{2}} \frac{\mathbf{h}_{2}^{H} \mathbf{Z} \mathbf{h}_{2}}{\sqrt{\left(\mathbf{h}_{2}^{H} \mathbf{Z}^{H} \mathbf{Z} \mathbf{h}_{2}\right)}} .
$$

If the matrix $\mathbf{Z}$ is a projection matrix (Equation (15)), it is idempotent, i.e., $\mathbf{Z}=\mathbf{Z}^{2}$ [25]. We can then write $\mathbf{h}_{2}^{H} \mathbf{Z}^{H} \mathbf{Z} \mathbf{h}_{2}=\mathbf{h}_{2}^{H} \mathbf{Z} \mathbf{h}_{2}$ and $\mathbf{h}_{2}^{H} \mathbf{w}_{2}=\sqrt{P_{2}\left(\mathbf{h}_{2}^{H} \mathbf{Z} \mathbf{h}_{2}\right)}$ Next, applying the singular-value decomposition to the matrix $\mathbf{Z}$ we obtain $\mathbf{h}_{2}^{H} \mathbf{Z} \mathbf{h}_{2}=\mathbf{h}_{2}^{H} \mathbf{U} \mathbf{\Lambda} \mathbf{U}^{H} \mathbf{h}_{2}$. The matrix $\mathbf{U}$ is a unitary matrix of eigenvectors and $\boldsymbol{\Lambda}$ is a diagonal matrix containing the eigenvalues. Because, the properties of a zero-mean complex Gaussian vector do not change when multiplied with a unitary matrix, we have $\mathbf{h}_{2}^{H} \mathbf{U} \sim \mathbf{h}_{2}^{H}$. From the results above we obtain

$$
E\left[\mathbf{h}_{2}^{H} \mathbf{w}_{2}\right]=E\left[\sqrt{P_{2}\left(\mathbf{h}_{2}^{H} \mathbf{\Lambda}_{w} \mathbf{h}_{2}\right)}\right] .
$$

Again, the matrix $\mathbf{Z}$ being idempotent, its eigenvalues are either 1 or 0 [25]. As a result, the rank of $\mathbf{Z}$ equals its trace

$$
\begin{aligned}
\operatorname{rank}(\mathbf{Z}) & =\operatorname{tr}\left(\mathbf{I}_{N_{t}}-\mathbf{h}_{c l}\left(\mathbf{h}_{c l}^{H} \mathbf{h}_{c l}\right)^{-1} \mathbf{h}_{c l}^{H}\right) \\
& =\operatorname{tr}\left(\mathbf{I}_{N_{t}}\right)-\operatorname{tr}\left(\mathbf{h}_{c l}\left(\mathbf{h}_{c l}^{H} \mathbf{h}_{c l}\right)^{-1} \mathbf{h}_{c l}^{H}\right)=N_{t}-1 .
\end{aligned}
$$

The term $E\left[\mathbf{h}_{2}^{H} \mathbf{w}_{2}\right]$ can then equivalently be expressed as

$$
E\left[\mathbf{h}_{2}^{H} \mathbf{w}_{2}\right]=E\left[\sqrt{P_{2} \sum_{n=1}^{N t-1}\left|\mathbf{h}_{2}^{n}\right|^{2}}\right] .
$$

From the equation (20) we can write the term $E\left[\left(\mathbf{h}_{2}^{H} \mathbf{w}_{2}\right)^{2}\right]$ as

$$
\left[\begin{array}{lll}
\Sigma & - & 1
\end{array}\right]
$$

The expression $\left|\mathbf{h}_{2}^{n}\right|^{2}$ follows a Chi-square distribution [24], we hence obtain

$$
E\left[\sum_{n=1}^{N_{t}-1}\left|\mathbf{h}_{2}^{n}\right|^{2}\right]=\frac{\Gamma\left(N_{t}\right)}{\left(N_{t}-2\right) !}=N_{t}-1
$$

where $\Gamma$ denotes the Gamma function. Combining the results above to the ergodic capacity of the network with basic access $\left(C_{E}\right)$ combined with ZF spatial-reuse spatial-reuse gives $\left(C_{\mathrm{ZF}}\right)$ 


$$
\begin{aligned}
& \left(\bar{\sigma} \sum^{-}\left[\begin{array}{ll}
\mid & \mid
\end{array}\right]\right) \\
& \left(\bar{\sigma} \sum^{-}\left[\begin{array}{ll}
\mid & \mid
\end{array}\right]\right)
\end{aligned}
$$

For example, for the scenario given above (4-B) the ergodic capacity with ZF beamforming scheme is

$$
C_{\mathrm{ZF}}=C_{E}+\log _{2}\left(1+100\left(N_{t}-1\right)\right) 0.132+\log _{2}\left(1+100\left(N_{t}-1\right)\right) 0.167 .
$$$$
=13+2=15 \mathrm{bps} / \mathrm{Hz}
$$

This represents a $15.4 \%$ improvement of the network capacity.

\section{Spatial-reuse with SJNR beamforming}

This section presents the SJNR beamformer based on the result previously proposed in [12] and [26]. The SJNR beamformer exploits the knowledge of the local channels to maximize the SINR criterion at both receiving STAs. Based on Equations (5) and (6), the SINR at the Relay ${ }_{1}$ and Relay 2 is

$$
\operatorname{SINR}_{1}=\frac{\left|\mathbf{h}_{1}^{H} \mathbf{w}_{1}\right|^{2}}{\left|\mathbf{h}_{c l}^{H} \mathbf{w}_{2}\right|^{2}+\sigma_{n}^{2}} \quad \text { and } \quad \operatorname{SINR}_{2}=\frac{1}{\sigma_{n}^{2}} \mid \mathbf{h}_{2}^{H} \mathbf{w}_{2}(25)
$$

Finding the beamforming vectors $\mathbf{w}_{1}$ and $\mathbf{w}_{2}$ that maximize the individual SINRs, or their sum, requires the knowledge of the channels and beamforming vectors. That is, the value of $\operatorname{SINR}_{1}$ depends also on the beamforming vector $\mathbf{w}_{2}$ and $\mathbf{h}_{c l}$. This is challenging to implement in an IEEE802.11s network as joint beamforming is necessary and a centralized processor must compute the beamforming weights. To circumvent this, we define the following objective function that is proportional to the total system capacity (in bit per second per $\mathrm{Hz}$ ) for a sufficiently high SINR

B denotes the bandwidth (in $\mathrm{Hz}$ ). From (26) we can formulate the objective function as

$$
\begin{aligned}
\max _{\mathbf{w}_{1}, \mathbf{w}_{2}} \log _{2}\left(\operatorname{SINR}_{1} \times \operatorname{SINR}_{2}\right) & =\max _{\mathbf{w}_{1}, \mathbf{w}_{2}} \frac{\left|\mathbf{h}_{1}^{H} \mathbf{w}_{1}\right|^{2}\left|\mathbf{h}_{2}^{H} \mathbf{w}_{2}\right|^{2}}{\left.\left(\left|\mathbf{h}_{c l}^{H} \mathbf{w}_{2}\right|^{2}\right)+\sigma_{n}^{2}\right)\left(\sigma_{n}^{2}\right)} \\
& =\max _{\mathbf{w}_{1}}\left|\mathbf{h}_{1}^{H} \mathbf{w}_{1}\right|^{2} \times \max _{\mathbf{w}_{2}} \frac{\left|\mathbf{h}_{2}^{H} \mathbf{w}_{2}\right|^{2}}{\left|\mathbf{h}_{c l}^{H} \mathbf{w}_{2}\right|^{2}+\sigma_{n}^{2}} .
\end{aligned}
$$

This shows that the optimizations of $\mathbf{w}_{2}$ can be done independently

$$
\mathbf{w}_{2}^{\text {opt }}=\max _{\mathbf{w}_{2}} \frac{\left|\mathbf{h}_{2}^{H} \mathbf{w}_{2}\right|^{2}}{\left|\mathbf{h}_{c l}^{H} \mathbf{w}_{2}\right|^{2}+\sigma_{n}^{2}} .
$$

Defining $\mathbf{w}_{2}^{H} \mathbf{w}_{2}=P_{2}\left(\mathbf{w}_{2}^{N}\right)^{H} \mathbf{w}_{2}^{N}$, where $\left(\mathbf{w}_{2}^{N}\right)^{H} \mathbf{w}_{2}^{N}=1$ we can express Equation (28) as

$$
\mathbf{w}_{2}^{\mathrm{opt}}=\sqrt{P_{2}} \max _{\mathbf{w}_{2}^{N}} \frac{P_{2}\left|\mathbf{h}_{2}^{H} \mathbf{w}_{2}^{N}\right| 2}{P_{2}\left|\mathbf{h}_{c l}^{H} \mathbf{w}_{2}^{N}\right|^{2}+\sigma_{n}^{2}}=\sqrt{P_{2}} \max _{\mathbf{w}_{2}^{N}} \frac{\left|\mathbf{h}_{2}^{H} \mathbf{w}_{2}^{N}\right| 2}{\left|\mathbf{h}_{c l}^{H} \mathbf{w}_{2}^{N}\right|^{2}+\frac{\sigma_{n}^{2}}{P_{2}}} .
$$

In such a case, maximizing the capacity does not require any collaboration between the transmitters. The beamformer at Relay 2 exploits the knowledge of its local channels only and does not depend on the beamforming vector at the other transmitter. The factor in (29) can be recognized as generalized Rayleigh quotient problems whose solution is given in [25]. The beamforming vectors based on the objective functions above can be expressed as

$$
\mathbf{w}_{2}^{\mathrm{opt}}=\sqrt{P_{2} e_{v}}\left(\left(\mathbf{h}_{c l} \mathbf{h}_{c l}^{H}+\sigma_{n}^{2}\right)^{-1} \mathbf{h}_{2} \mathbf{h}_{2}^{H}\right)
$$

where $e_{v}(\mathbf{A})$ denotes the eigenvector corresponding to the largest eigenvalue of matrix $\mathbf{A}$ and thus fulfill the power constraint in (4). In (28), the proposed beamformer exploits the knowledge of the local channels to find the best trade-off to optimize the SINR criterion between maximizing the energy of the useful information (transmit-MRC), i.e., the terms at the numerator, and minimizing the interference terms (ZF), i.e., the terms at the denominator.

Because the computation of the beamforming vector $\mathbf{w}_{2}^{\text {opt }}$ is based on an eigenvalue decomposition it is challenging to obtain a close-form solution of the ergodic capacity. As a result, we approximate the capacity gain of the SJNR beamformer through simulations. Section 5 presents the results.

\section{E. Generalization to multiple concurrent transmissions}

While we have shown how to implement spatial reuse in an IEEE 802.11n wireless mesh network, the considered setup (and the proposed derivations) can be extended to the case with more than two concurrent transmissions. A third Relay may transmit concurrently in addition to the primary user (Relay ${ }_{1}$ ) and the first concurrent Relay $\left(\right.$ Relay $\left._{2}\right)$. As for the Relay 2 , this is possible if the Relay 3 has more antennas than the intended receiver and if Relay $_{3}$ does not interfere with both intended receivers from Relay ${ }_{1}$ and Relay ${ }_{2}$, i.e., $\mathrm{STA}_{1}$ and $\mathrm{STA}_{2}$, respectively. For example if $\mathrm{STA}_{2}$ is outside its coverage range or if Relay ${ }_{3}$ is equipped with enough antennas to cancel interfere towards both $\mathrm{STA}_{1}$ and $\mathrm{STA}_{2}$. If such requirements are fulfilled, the Relay $_{3}$ also transmits on the same time and frequency resources as the Relay ${ }_{1}$ and Relay $_{2}$, hence providing a further increase in network capacity.

While several non-interfering transmissions could be scheduled, such asymptotic analysis that neglect the practical constraints of such a setup, e.g., delay 
constraints for the coordination of the transmissions, could be interesting to establish theoretical bounds on spatial reuse, but are in our opinion beyond the scope of this paper.

\section{Results}

The results in this section provide the ergodic and the simulated performance of the schemes of interest (Section 4) and verify the analytical results. The specific scenario that we consider for the performance analysis is an IEEE802.11s network composed of two relaying stations close to the access point and hence source of most of the traffic. Since they are close to the access point, the relays are also close to each other, hence blocking each other's channel access when transmitting. Simulation results of the capacity are shown for the various schemes in a given scenario and a varying SNR (Section 5-A). Section 5-B discusses the impact of the size of the overlapping area on the performance of the various schemes. The analytical results of the ergodic capacity (Section 4) are verified and compared with the simulated results in Section 5-C.

\section{A. Capacity gain of the various schemes}

Figure 6 displays the capacity improvements (in percent) of the ZF and SJNR beamformers over the IEEE802.11s basic access scheme for a varying SNR value. The scenario of interest is as follows, we assume a noise floor of $-85 \mathrm{dBm}$ (for a $20 \mathrm{MHz}$ channel bandwidth), the coverage radius of the relay STAs are $r_{1}=r_{2}=100 \mathrm{~m}$ and the distance between them is $d=60$ and $d=100 \mathrm{~m}$. The cross channels have a variance of $\sigma_{c l}^{2}=0.3$ and each relay STA is equipped with two transmit antennas $\left(N_{t}=\right.$ 2 ). We simulate the varying of the SNR by adapting the transmit (hence receive) power of the relay STAs, e.g., a SNR of $0 \mathrm{~dB}$ indicates a receive power at the STA of $-85 \mathrm{dBm}$, similarly a SNR of $30 \mathrm{~dB}$ gives $-55 \mathrm{dBm}$ at the STA. Because we vary the transmit power, we adapt the carrier sensing threshold accordingly to keep the coverage radius of the relays unchanged.

From this Figure, we can observe that the SJNR beamformer outperforms the ZF beamformer in the low SNR region $(<15 \mathrm{~dB})$ while achieving the same performance at high SNR. At low SNR, the noise is the major source of impairment, mitigating the interference term is hence not optimal. Because the SJNR beamformer makes a trade-off between mitigating the interference term and maximizing the energy towards the concurrent STA, it outperforms the ZF beamformer in the low SNR region. In the high SNR region, the interference term becomes the main source of errors and canceling the interference term becomes now optimal, i.e., both the SJNR and ZF beamformers achieve then similar performance. Moreover, as the distance (d) between relay STAs reduces, the overlapping area increases, resulting in a higher number of blocked transmissions and more

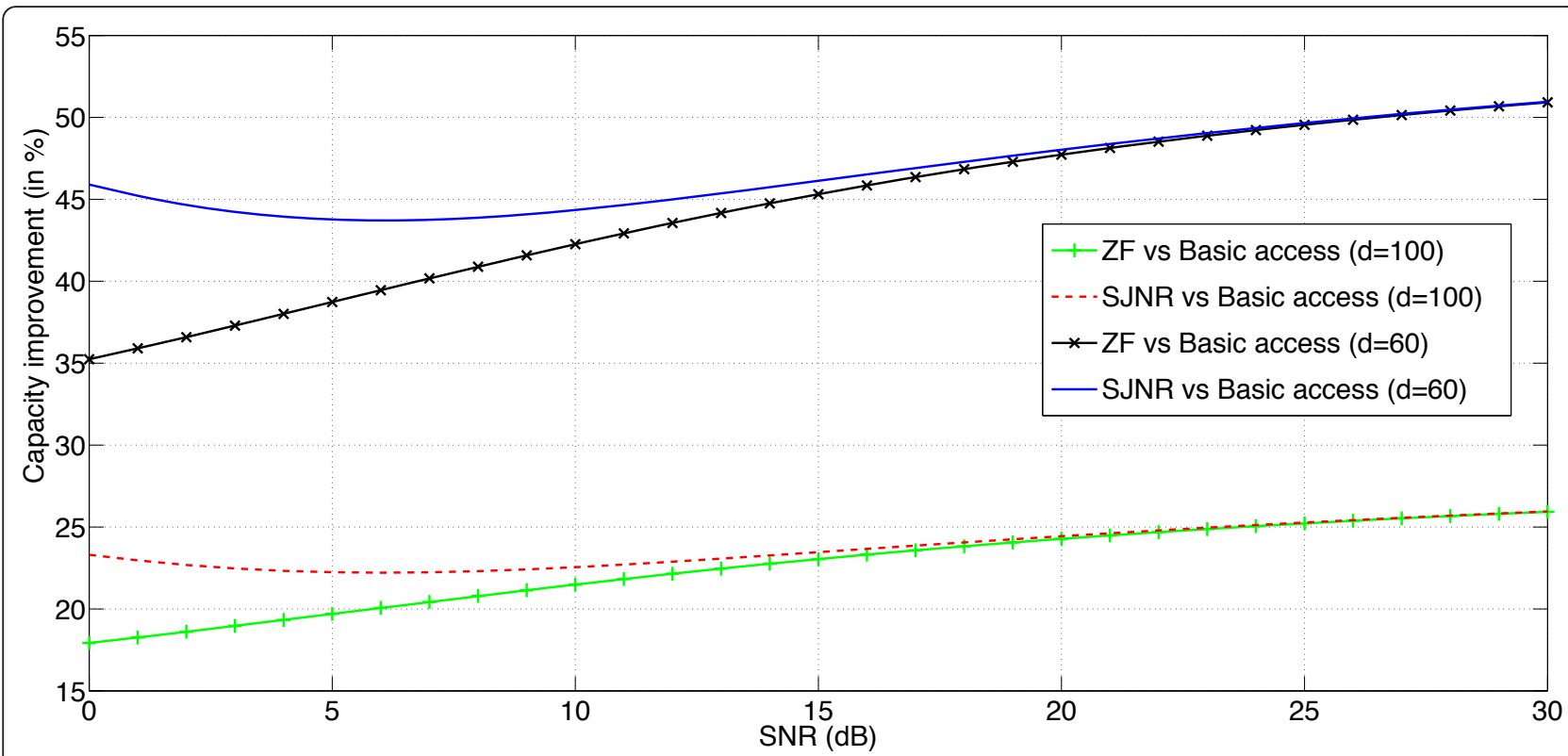

Figure 6 Capacity gain improvement from the zero-forcing (ZF) and signal-to-jamming noise ratio (SJNR) beamformers over the basic IEEE802.11s access scheme. The results are shown for a varying signal-to-noise ratio (SNR) and for a distance $d=60 \mathrm{~m}$ and $d=100 \mathrm{~m}$ between the Relays. The curves ZF Vs. Basic access and SJNR vs. Basic access indicate the capacity improvement of the ZF and SJNR beamformers over the basic IEEE802.11s access scheme. In this situation, both the SJNR and ZF beamformers show significant capacity gain improvement compared to the basic access scheme. Moreover, the SJNR outperforms the ZF beamformers in the low-SNR region $(<15 \mathrm{~dB})$. 
opportunities to gain from using beamforming techniques. Consequently, the capacity improvement for both the ZF and SJNR beamformer over the basic scheme is higher at $d=60$ than for $d=100$.

\section{B. Impact of the overlapping area}

Figure 7 displays the capacity of the ZF and SJNR beamformers and the IEEE802.11s basic access scheme when varying the distance $(d)$ between the two relay STAs. In Section 5-A, we observed that the distance between the relays impacts the performance of the network, i.e., reducing this distance will enlarge the size of the overlapping area; consequently increasing the probability of blocked transmissions (Section 3-A). However, this negative impact can be mitigated using the ZF and SJNR techniques we propose.

For the simulation scenario, we assume a fixed transmit power and display the results for a $5 \mathrm{~dB}$ SNR, the radius of the relay STAs are $r_{1}=r_{2}=100 \mathrm{~m}$, the crosschannels have a variance of $\sigma_{c l}^{2}=0.3$ and each relay STA is equipped with two transmit antennas $\left(N_{t}=2\right)$. We then vary the distance between the relay STAs, from $d=0$ (i.e., full overlapping) to $d=200 \mathrm{~m}$ (i.e., no overlapping). At $d=200 \mathrm{~m}$, there is no overlapping hence no blocked transmissions and the various schemes achieve then the same capacity. However, Figure 7 shows that as the distance between the relay STAs decreases the network capacity of all schemes degrades. Nevertheless, these results show also that employing the
ZF and SJNR beamforming techniques mitigates this decrease in capacity and that the SJNR provides significant higher capacity gain compared to the basic access and the ZF schemes.

\section{Tightness of the analytical results}

Figure 8 displays the simulated and ergodic capacity of the ZF and the IEEE802.11s basic access schemes for a varying SNR value. We assume a noise floor of $-85 \mathrm{dBm}$ (for a $20 \mathrm{MHz}$ channel bandwidth), the coverage radius of the relay STAs are $r_{1}=r_{2}=100$ and the distance between them is $d=60$. The cross channels have a unit-variance of $\sigma_{c l}^{2}=0.3$ and each relay STA is equipped with two transmit antennas $\left(N_{t}=2\right)$. As for the setup in Section 5-A, we simulate the varying of the SNR by adapting the transmit (hence receive) power of the relay STAs and adapt the carrier sensing threshold accordingly to keep the coverage radius of the relays unchanged.

\section{Conclusion}

We present how beamforming techniques can be implemented on top of the IEEE802.11s MAC protocol, using the CSI and timing information readily available from the MCCA mechanism, to mitigate the CCI and increase the number of spatial-reuse opportunities; consequently maximizing the QoS of such networks by improving the capacity and latency. In addition, we propose the SJNR beamformer and show that it significantly

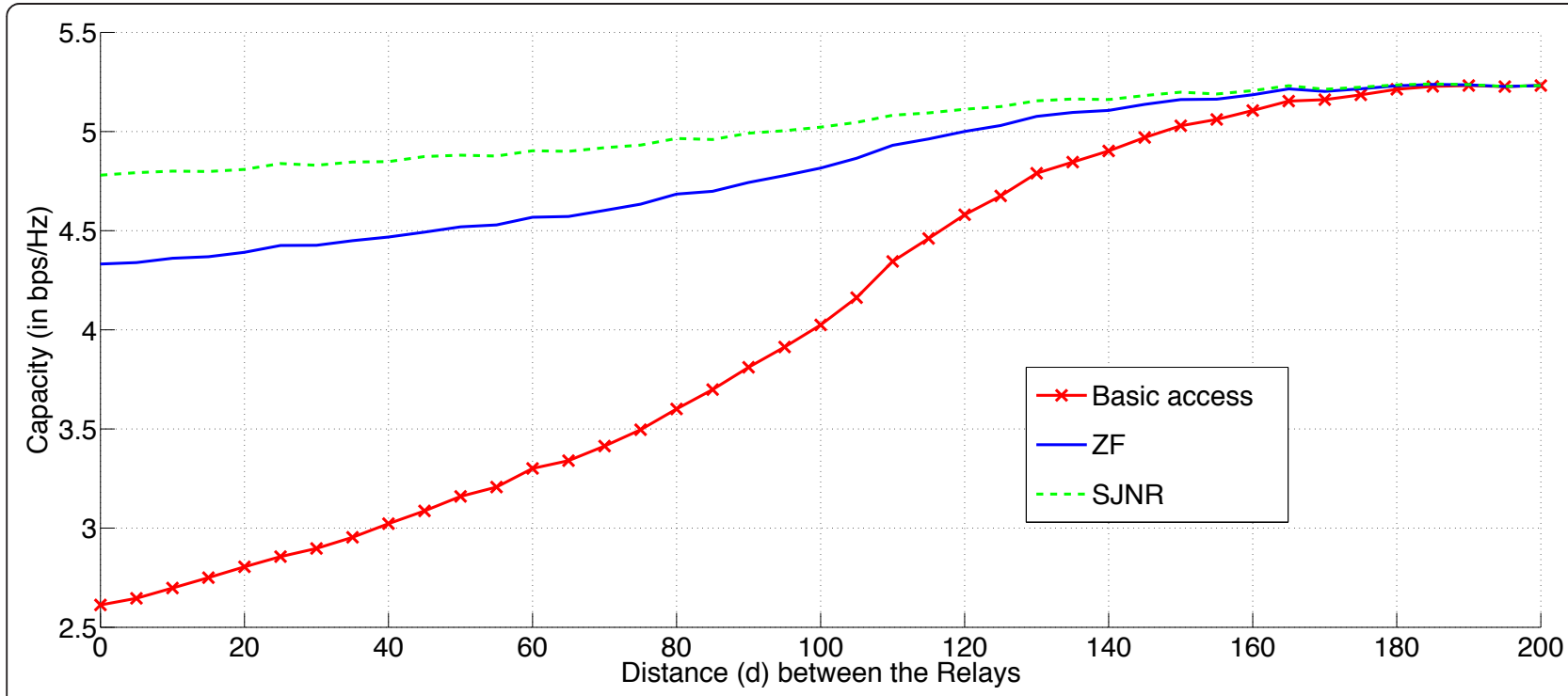

Figure 7 Impact of the overlapping area on the capacity of the network. The results are shown for a fixed SNR of $5 \mathrm{~dB}$ and for a varying distance $d$ between the Relays. The curve Basic access denotes the capacity of the basic IEEE802.11s access, i.e., when blocked transmissions are not mitigated. The curves ZF and SJNR represent the capacity gain obtained from these schemes (Section 4). We can observe that reducing the distance between the two relay STAs degrades the capacity of the network. However, this negative impact can be mitigated through the ZF and SJNR techniques we propose. Moreover, the SJNR beamformer outperforms the ZF scheme by more than 10\% and the basic IEEE802.11s access scheme by up to $85 \%$. 


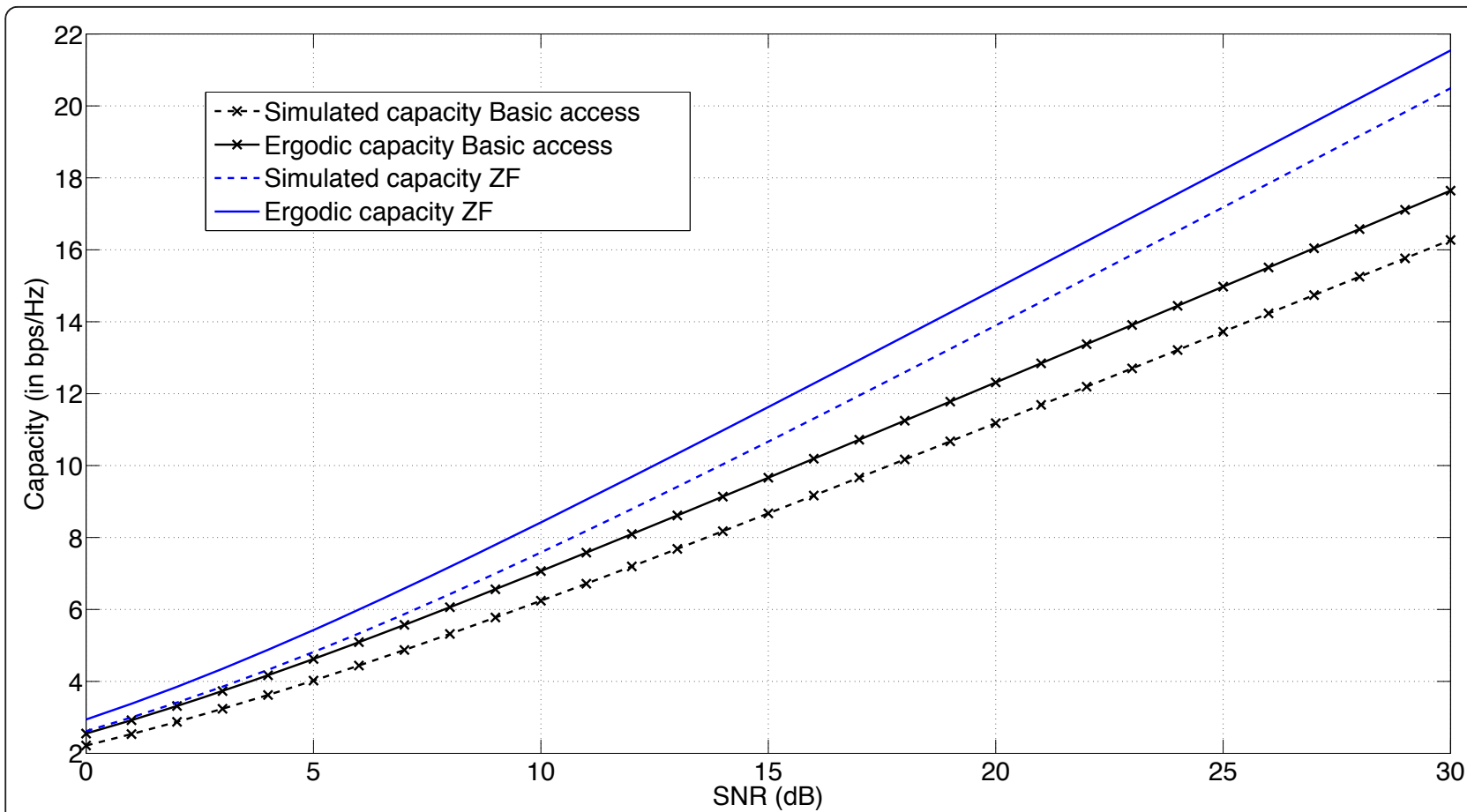

Figure 8 This figure shows the ergodic capacity (Section 4) and simulated capacity curves for the ZF and 802.11s basic access schemes for a varying SNR value. The curves Basic access and ZF denote the ergodic and simulated capacity of the basic IEEE802.11s access and zero-forcing schemes. The ergodic capacity provides an upper bound of the capacity and the results displayed in this figure shows that the proposed derivations follow the simulated results.

improves the spatial-reuse gain compared to the simple ZF beamformer and the basic IEEE $802.11 \mathrm{~s}$ access scheme. The ergodic capacity of the ZF beamformer and the basic IEEE802.11s access scheme is derived to analytically measure the gain of employing beamforming techniques in IEEE802.11s networks. The derivations are verified and compared with results from the simulations and we show that improvements of up to $\mathbf{8 5} \%$ are achieved as function of the scenario simulated and the beamforming technique used.

\section{Endnotes}

${ }^{a}$ The general expression of the capacity is usually given as follows, $C=B\left[\log _{2}\left(1+\operatorname{SINR}_{1}\right)+\log _{2}\left(1+\operatorname{SINR}_{2}\right)\right]$

\section{Author details}

1 Interuniversity Micro-Electronics Center (IMEC), Kapeldreef 75, 3001 Leuven, Belgium ${ }^{2}$ Department of Electrical Engineering, Katholieke Universiteit Leuven, Kasteelpark Arenberg 10, 3001 Leuven, Belgium ${ }^{3}$ Université Libre de Bruxelles, Avenue F. D. Roosevelt 50, 1050 Brussels, Belgium ${ }^{4}$ Nanjing University, HanKou Road 22, Nanjing 210093, P. R. China

\section{Competing interests}

The authors declare that they have no competing interests.

Received: 18 February 2011 Accepted: 22 October 2011 Published: 22 October 2011

\section{References}

1. IEEE, "IEEE draft standard for information technology-telecommunications and information exchange between systems-local and metropolitan area networks- specific requirements-part 11: Wireless LAN medium access control (MAC) and physical layer (PHY) specifications-amendment 10: Mesh networking". IEEE P802.11s/D10.0, March 2011 (Mar. 2011)

2. IEEE, "IEEE draft recommended practice for information technologytelecommunications and information exchange between systems- local and metropolitan area networks-specific requirements part 15.5: Mesh topology capability in wireless personal area networks (WPANs)". IEEE Unapproved Draft Std P802.15.5/D7 (Oct. 2008)

3. IEEE, "IEEE standard for local and metropolitan area networks part 16: Air interface for fixed and mobile broadband wireless access systems amendment 2: Physical and medium access control layers for combined fixed and mobile operation in licensed bands and corrigendum 1". IEEE Std 802.16e-2005 and IEEE Std 802.16-2004/Cor 1-2005 (Amendment and Corrigendum to IEEE Std 802.16-2004), 0-822 (2006)

4. J Ben-Othman, L Mokdad, MO Cheikh, "A new architecture of wireless mesh networks based IEEE 802.11s directional antennas", in Proc IEEE ICC (Jun. 2011)

5. M Timmers, Distributed Control of Software-Defined Radios using Flexible Spectrum Access (Katholieke Universiteit Leuven - Faculty of Engineering 2009)

6. T Hidekuma, G Hasegawa, M Sasabe, H Nakano, "Degree-based power control method for increasing spatial reuse in TDMA-based wireless mesh networks", in Proc ICN, pp. 121-126 (March 2009)

7. H Ma, R Vijayakumar, S Roy, J Zhu, "Optimizing 802.11 wireless mesh networks based on physical carrier sensing", in Networking, IEEE/ACM Transactions on. 17(5), 1550-1563 (Oct. 2009)

8. B Alawieh, Y Zhang, C Assi, H Mouftah, "Improving spatial reuse in multihop wireless networks - a survey". IEEE Communications Surveys Tutorials 11(3), 71-91 (2009)

9. J El-Najjar, H AlAzemi, C Assi, "On the interplay between spatial reuse and network coding in wireless networks". IEEE Transactions on Wireless Communications 10(2), 560-569 (February 2011) 
10. M Timmers, S Pollin, A Dejonghe, L Van der Perre, F Catthoor, "Throughput modeling of large-scale 802.11 networks", in Proc IEEE Globecom (Dec. 2008)

11. S-H Park, H Park, I Lee, "Beamforming design based on virtual SINR maximization for interference networks", in Proc IEEE ICC (Jun. 2011)

12. R Zakhour, D Gesbert, "Distributed multicell-MISO precoding using the layered virtual SINR framework". IEEE Transactions on Wireless Communications 9(8), 2444-2448 (Aug. 2010)

13. K Balachandran, J Kang, K Karakayali, K Rege, "NICE: A network interference cancellation engine for opportunistic uplink cooperation in wireless networks". IEEE Transactions on Wireless Communications 10(2), 540-549 (Feb. 2011)

14. K Huang, J Andrews, R Heath, D Guo, R Berry, "Spatial interference cancellation for mobile ad hoc networks: Perfect CSI", in Proc IEEE Globecom, pp. 1-5 (Dec. 2008)

15. K Fakih, J-F Diouris, G Andrieux, "Beamforming in ad hoc networks: MAC design and performance modeling". EURASIP Journal on Wireless Communications and Networking 15 (2009)

16. J Winters, "Smart antenna techniques and their application to wireless ad hoc networks". IEEE Wireless Communications Magazine 13(4), 77-83 (Aug. 2006). doi:10.1109/MWC.2006.1678168

17. T Korakis, G Jakllari, L Tassiulas, "CDR-MAC: A protocol for full exploitation of directional antennas in ad hoc wireless networks". IEEE Transactions on Mobile Computing 7(2), 145-155 (Feb. 2008)

18. A Nasipuri, S Ye, J You, R Hiromoto, "A MAC protocol for mobile ad hoc networks using directional antennas", in Proc IEEE WCNC. 3, 1214-1219 (2000)

19. N Fahmy, T Todd, V Kezys, "Ad hoc networks with smart antennas using IEEE 802.11-based protocols", in Proc IEEE ICC. 5, 3144-3148 (2002)

20. T Nadeem, "Analysis and enhancements for IEEE 802.11 networks using directional antenna with opportunistic mechanisms". IEEE Transactions on Vehicular Technology 59(6), 3012-3024 (July 2010)

21. M Sawahashi, Y Kishiyama, A Morimoto, D Nishikawa, M Tanno, "Coordinated multipoint transmission/reception techniques for LTEadvanced, coordinated and distributed OFDM". IEEE Transactions on Wireless Communications 17(3), 26-34 (Jun. 2010)

22. A Paulraj, R Nabar, D Gore, Introduction to Space-Time Wireless Communications (Cambridge University Press, 2008)

23. A Goldsmith, Wireless Communications (Cambridge University Press, 2005)

24. MK Simon, Probability Distributions Involving Gaussian Random Variables (Springer, 2006)

25. RA Horn, CR Johnson, Matrix Analysis (Cambridge University Press, 1985)

26. Y Lebrun, J Nsenga, V Ramon, A Bourdoux, F Horlin, R Lauwereins, "Maximum SINR-based beamforming for the MISO interference channel", in Proc EUSIPCO, pp. 779-783 (Sep. 2009)

doi:10.1186/1687-1499-2011-136

Cite this article as: Lebrun et al: Beamforming techniques for enabling spatial-reuse in MCCA 802.11s networks. EURASIP Journal on Wireless Communications and Networking 2011 2011:136.

\section{Submit your manuscript to a SpringerOpen ${ }^{\circ}$ journal and benefit from:}

- Convenient online submission

- Rigorous peer review

- Immediate publication on acceptance

- Open access: articles freely available online

- High visibility within the field

- Retaining the copyright to your article

Submit your next manuscript at $\gg$ springeropen.com 